\title{
PERTURBATION BOUNDS FOR ISOTROPIC INVARIANT SUBSPACES OF SKEW-HAMILTONIAN MATRICES*
}

\author{
DANIEL KRESSNER ${ }^{\dagger}$
}

\begin{abstract}
We investigate the behavior of isotropic invariant subspaces of skew-Hamiltonian matrices under structured perturbations. It is shown that finding a nearby subspace is equivalent to solving a certain quadratic matrix equation. This connection is used to derive meaningful error bounds and condition numbers that can be used to judge the quality of invariant subspaces computed by strongly backward stable eigensolvers.
\end{abstract}

Key words. skew-Hamiltonian, invariant subspace, perturbation analysis, Sylvester equation, Riccati equation

AMS subject classifications. 47A15, 47A55, 65F 15

1. Introduction. A real $2 n \times 2 n$ matrix of the form

$$
W=\left[\begin{array}{cc}
A & G \\
H & A^{T}
\end{array}\right], \quad G=-G^{T}, \quad H=-H^{T},
$$

with $A, G, H \in \mathbb{R}^{n \times n}$ is called skew-Hamiltonian. The imposed structure has a number of consequences for the eigenvalues and eigenvectors of $W$; one is that each eigenvalue appears at least twice. Hence, well-known results from matrix perturbation theory predict that the eigenvectors of $W$ are extremely ill-conditioned, i.e., they may change drastically under small perturbations. For example, consider the parameterdependent matrix

$$
W\left(\varepsilon_{1}, \varepsilon_{2}\right)=\left[\begin{array}{cccc}
1 & 0 & 0 & 0 \\
0 & 2 & 0 & 0 \\
\varepsilon_{1} & \varepsilon_{2} & 1 & 0 \\
-\varepsilon_{2} & 0 & 0 & 2
\end{array}\right]
$$

The vector $e_{1}=[1,0,0,0]^{T}$ is an eigenvector of $W(0,0)$ associated with the eigenvalue $\lambda=1$. No matter how small $\varepsilon_{1}>0$ is, any eigenvector of $W\left(\varepsilon_{1}, 0\right)$ associated with $\lambda$ has the completely different form $[0,0, \alpha, 0]^{T}$ for some $\alpha \neq 0$. On the other hand, $W\left(0, \varepsilon_{2}\right)$ has an eigenvector $\left[1,0,0, \varepsilon_{2}\right]^{T}$ rather close to $e_{1}$. The fundamental difference between $W\left(\varepsilon_{1}, 0\right)$ and $W\left(0, \varepsilon_{2}\right)$ is that the latter is a skew-Hamiltonian matrix while the former is not.

In this paper we investigate the behavior of eigenvectors of skew-Hamiltonian matrices under perturbations that are structure-preserving, as in the case of $W\left(0, \varepsilon_{2}\right)$. More general, the discussion is concerned with isotropic invariant subspaces, those are - loosely speaking - the invariant subspaces of $W$ associated with at most one copy of each eigenvalue. We derive error bounds that allow users of strongly backward stable eigensolvers $[11,19]$ to quantify their obtained results. Furthermore, applications that directly depend on the computation of isotropic invariant subspaces such as certain Riccati equations [13] and quadratic eigenvalue problems [18] may benefit from these bounds.

\footnotetext{
*Supported by the DFG Research Center "Mathematics for Key Technologies" (FZT 86) in Berlin and a Marie Curie fellowship in the frame of the Control Training Site (MCFI-2001-00403).

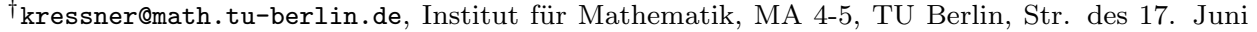
136, D-10623 Berlin, FRG.
} 
The perturbation theory given here owes much to the fact that there exist considerably simple condensed forms for skew-Hamiltonian matrices. Section 2 reviews some of these forms along with other theoretical tools required later on. In Section 3 , the connection between finding a nearby isotropic subspace and solving a quadratic matrix equation is explained. The solution of this equation is complicated by an artificial singularity, its lengthy derivation is described in Section 4. The subsequent section contains the central result of this work, Theorem 5.2 gives an upper bound for the sensitivity of isotropic invariant subspaces. This will lead us to define a corresponding condition number, and Section 6 contains some discussion on how this quantity can be computed. Finally, in Section 7 a numerical example is presented to illustrate the use of the derived condition number.

2. Basic tools. Equivalent to the block representation (1.1) a skew-Hamiltonian matrix $W$ is characterized by the fact that $J_{n} W$ is skew-symmetric, where $J_{n}=$ $\left[\begin{array}{cc}0 & I_{n} \\ -I_{n} & 0\end{array}\right]$ and $I_{n}$ is the $n \times n$ identity matrix. In the following we will drop the subscript $n$ whenever the dimension of the corresponding matrix is clear from its context. A matrix $S \in \mathbb{R}^{2 n \times 2 n}$ is called symplectic if $S^{T} J S=J$. It is easy to show that in this case $J S^{-1} W S$ is skew-symmetric, thus symplectic similarity transformations preserve skew-Hamiltonian structures. Moreover, an orthogonal matrix $U$ is symplectic if and only if it has the representation

$$
U=\left[\begin{array}{cc}
U_{1} & U_{2} \\
-U_{2} & U_{1}
\end{array}\right], \quad U_{1}, U_{2} \in \mathbb{R}^{n \times n} .
$$

We will call such a matrix orthogonal symplectic. An important property of $U$ is that its first $k \leq n$ columns span an isotropic subspace.

Definition 2.1. A subspace $\mathcal{X} \subset \mathbb{R}^{2 n}$ is called isotropic if $J \mathcal{X} \perp \mathcal{X}$.

Van Loan [19] showed that for any skew-Hamiltonian matrix $W$ there exists of an orthogonal symplectic matrix $U$ so that

$$
U^{T} W U=\left[\begin{array}{cc}
\tilde{A} & \tilde{G} \\
0 & \tilde{A}^{T}
\end{array}\right]
$$

where $\tilde{A}$ is in real Schur form. Moreover, real eigenvalues and complex conjugate pairs of eigenvalues may appear in any desirable order on the diagonal of $\tilde{A}$. Closely related to (2.2) is the following characterization of isotropic invariant subspaces of $W$.

LEMMA 2.2. Let $W \in \mathbb{R}^{2 n \times 2 n}$ be a skew-Hamiltonian matrix and let $X \in \mathbb{R}^{2 n \times k}$ $(k \leq n)$ have orthonormal columns. Then the columns of $X$ span an isotropic invariant subspace of $W$ if and only if there exists an orthogonal symplectic matrix $U=\left[X, Z, J^{T} X, J^{T} Z\right]$ with some $Z \in \mathbb{R}^{2 n \times(n-k)}$ so that

$$
U^{T} W U=\begin{aligned}
& k \\
& k \\
& k \\
& k-k \\
& n-k
\end{aligned}\left[\begin{array}{cccc}
A_{11} & A_{12} & G_{11} & G_{12} \\
0 & A_{22} & -G_{12}^{T} & G_{22} \\
0 & 0 & A_{11}^{T} & 0 \\
0 & H_{22} & A_{12}^{T} & A_{22}^{T}
\end{array}\right] .
$$

Proof. Assume that the columns of $X$ span an isotropic subspace. Then the symplectic QR factorization [2] can be used to construct an orthogonal symplectic matrix $U=\left[X, Z, J^{T} X, J^{T} Z\right]$. Moreover, if the columns of $X$ span an invariant 
subspace then $\left[Z, J^{T} X, J^{T} Z\right]^{T} W X=0$, completing the proof of (2.3). The other direction is straightforward.

As the spectral properties of $A_{11}=X^{T} W X$ and

$$
\left[\begin{array}{ll}
A_{22} & G_{22} \\
H_{22} & A_{22}^{T}
\end{array}\right]=\left[Z, J^{T} Z\right]^{T} W\left[Z, J^{T} Z\right]
$$

do not depend on the choice of bases the following definition can be used to adapt the notion of simple invariant subspaces to skew-Hamiltonian matrices.

Definition 2.3. Let the orthonormal columns of $X \in \mathbb{R}^{2 n \times k}$ span an isotropic invariant subspace $\mathcal{X}$ of a skew-Hamiltonian matrix $W$. Furthermore, choose $Z \in$ $\mathbb{R}^{2 n \times(n-k)}$ so that $U=\left[X, Z, J^{T} X, J^{T} Z\right]$ is orthogonal symplectic and $U^{T} W U$ has the form (2.3). Then $\mathcal{X}$ is called semi-simple if $\lambda\left(A_{11}\right) \cap \lambda\left(\left[\begin{array}{ll}A_{22} & G_{22} \\ H_{22} & A_{22}^{T}\end{array}\right]\right)=\emptyset$ and $A_{11}$ is nonderogatory, i.e., each eigenvalue of $A_{11}$ has geometric multiplicity one.

Semi-simple subspaces allow us to block diagonalize $W$ by a simple transformation. For this purpose, we require two facts about Sylvester equations. The first is a well-known result, proofs can be found in many places, see e.g. [8].

Proposition 2.4. The Sylvester equation

$$
A P-P B=C
$$

with $A \in \mathbb{R}^{n \times n}, B \in \mathbb{R}^{m \times m}$ and $C \in \mathbb{R}^{n \times m}$ has a unique solution $P \in \mathbb{R}^{n \times m}$ if and only if $\lambda(A) \cap \lambda(B)=\emptyset$.

The second is concerned with a certain type of singular Sylvester equations that do not fit into the framework of Proposition 2.4.

Proposition 2.5. The Sylvester equation

$$
A P-P A^{T}=G
$$

is solvable for all skew-symmetric matrices $G$ if and only if $A$ is nonderogatory. In this case, any solution $P$ to (2.4) is real and symmetric.

Proof. This result can be found in [4]. Actually, the second part is not explicitely stated there but follows easily from the proof of Proposition 5 in [4].

Propositions 2.4 and 2.5 can be combined to successively annihilate the blocks $A_{12}, G_{12}$ and $G_{11}$ in the block representation (2.3) for a semi-simple subspace. To see this, solve

$$
A_{11}\left[\begin{array}{ll}
P_{1} & P_{2}
\end{array}\right]-\left[\begin{array}{ll}
P_{1} & P_{2}
\end{array}\right]\left[\begin{array}{ll}
A_{22} & G_{22} \\
H_{22} & A_{22}^{T}
\end{array}\right]=-\left[\begin{array}{ll}
A_{12} & G_{12}
\end{array}\right],
$$

and construct the symplectic matrix

$$
S_{P}=\left[\begin{array}{cccc}
I & P_{1} & -P_{1} P_{2}^{T} & P_{2} \\
0 & I & P_{2}^{T} & 0 \\
0 & 0 & I & 0 \\
0 & 0 & -P_{1}^{T} & I
\end{array}\right],
$$

yielding

$$
S_{P}^{-1}\left[\begin{array}{cccc}
A_{11} & A_{12} & G_{11} & G_{12} \\
0 & A_{22} & -G_{12}^{T} & G_{22} \\
0 & 0 & A_{11}^{T} & 0 \\
0 & H_{22} & A_{12}^{T} & A_{22}^{T}
\end{array}\right] S_{P}=\left[\begin{array}{cccc}
A_{11} & 0 & \tilde{G}_{11} & 0 \\
0 & A_{22} & 0 & G_{22} \\
0 & 0 & A_{11}^{T} & 0 \\
0 & H_{22} & 0 & A_{22}^{T}
\end{array}\right]
$$


with a skew-symmetric matrix $\tilde{G}_{11}$. Next, use a solution of

$$
A_{11} Q-Q A_{11}^{T}=-\tilde{G}_{11}
$$

to construct

$$
S_{Q}=\left[\begin{array}{cccc}
I & 0 & Q & 0 \\
0 & I & 0 & 0 \\
0 & 0 & I & 0 \\
0 & 0 & 0 & I
\end{array}\right]
$$

The matrix $S_{Q}$ is symplectic since Proposition 2.5 guarantees that $Q$ is symmetric. If the similarity transformation associated with $S_{Q}$ is applied to the right hand side of (2.5) then the block $\tilde{G}_{11}$ is annihilated. Note that there is a lot of freedom in the choice of $Q$ as equation (2.6) admits infinitely many solutions. From a numerical point of view the matrix $Q$ should be chosen so that the condition number of the product $S_{P} S_{Q}$ is as small as possible.

3. Perturbations and a quadratic matrix equation. Consider an isotropic invariant subspace $\mathcal{X}$ of a skew-Hamiltonian matrix $W$. Given a skew-Hamiltonian perturbation $E$ of small norm we now investigate the question whether $W+E$ has an isotropic invariant subspace $\hat{\mathcal{X}}$ close to $\mathcal{X}$. What follows is in many aspects similar to the treatment for general matrices by Stewart $[14,15]$, only that we end up with a quadratic matrix equation of quite a different nature.

Let the columns of $X$ form an orthonormal basis for $\mathcal{X}$. Apply Lemma 2.2 to construct a matrix $Y=\left[Z, J^{T} Z, J^{T} X\right]$ so that $\tilde{U}=[X, Y]$ is an orthogonal matrix. Note that $\tilde{U}^{T}(W+E) \tilde{U}$ is a permuted skew-Hamiltonian matrix and can be partitioned as

$$
\left.\tilde{U}^{T}(W+E) \tilde{U}=\begin{array}{ccc}
k & 2(n-k) & k \\
k & 2(n-k)
\end{array}\right]\left[\begin{array}{ccc}
W_{11} & W_{23}^{T} J_{n-k}^{T} & W_{13} \\
W_{21} & W_{22} & W_{23} \\
W_{31} & W_{21}^{T} J_{n-k} & W_{11}^{T}
\end{array}\right],
$$

where $W_{13}$ and $W_{31}$ are skew-symmetric matrices, and $W_{22}$ is skew-Hamiltonian. For $E=0$, the matrices $W_{21}$ and $W_{31}$ are zero and the other blocks in (3.1) correspond to the block representation (2.3) as follows:

$$
W_{11}=A_{11}, W_{13}=G_{11}, W_{22}=\left[\begin{array}{cc}
A_{22} & G_{22} \\
H_{22} & A_{22}^{T}
\end{array}\right], W_{23}=\left[\begin{array}{c}
-G_{12}^{T} \\
A_{12}^{T}
\end{array}\right] .
$$

Now, let

$$
\begin{aligned}
& \hat{X}=\left(X+Y\left[\begin{array}{l}
P \\
Q
\end{array}\right]\right)\left(I+P^{T} P+Q^{T} Q\right)^{-1 / 2} \\
& \hat{Y}=\left(Y-X\left[\begin{array}{ll}
P^{T} & Q^{T}
\end{array}\right]\right)\left(I+\left[\begin{array}{l}
P \\
Q
\end{array}\right]\left[\begin{array}{ll}
P^{T} & Q^{T}
\end{array}\right]\right)^{-1 / 2},
\end{aligned}
$$

where $P \in \mathbb{R}^{2(n-k) \times k}$ and $Q \in \mathbb{R}^{k \times k}$ are matrices to be determined so that $\hat{\mathcal{X}}=$ $\operatorname{span}(\hat{X})$ is an isotropic invariant subspace of $W+E$. This is equivalent to the conditions $Q^{T}-Q=P^{T} J P$ and $\hat{Y}^{T}(W+E) \hat{X}=0$. In terms of $(3.1)$, the latter can be written as

$$
\left[\begin{array}{l}
P \\
Q
\end{array}\right] W_{11}-\left[\begin{array}{cc}
W_{22} & W_{23} \\
W_{21}^{T} J & W_{11}^{T}
\end{array}\right]\left[\begin{array}{c}
P \\
Q
\end{array}\right]+\left[\begin{array}{c}
P \\
Q
\end{array}\right]\left[\begin{array}{c}
J W_{23} \\
W_{13}^{T}
\end{array}\right]^{T}\left[\begin{array}{l}
P \\
Q
\end{array}\right]=\left[\begin{array}{l}
W_{21} \\
W_{31}
\end{array}\right]
$$


Once we have solved (3.4) the sines of the canonical angles between $\mathcal{X}$ and $\hat{\mathcal{X}}$ are the singular values of

$$
Y^{T} \hat{X}=\left[\begin{array}{c}
P \\
Q
\end{array}\right]\left(I+P^{T} P+Q^{T} Q\right)^{-1 / 2}
$$

see e.g. [16, Sec. I.5]. We will see that (3.4) may admit infinitely many solutions satisfying $Q^{T}-Q=P^{T} J P$. In the interest of a small distance between $\mathcal{X}$ and $\hat{\mathcal{X}}$ a solution of small norm should be preferred.

4. A solution of the quadratic matrix equation. Solving (3.4) is complicated by two facts. First, we have to guarantee that the solution satisfies $Q^{T}-Q=$ $P^{T} J P$ and second, the linear part of (3.4) is close to a singular linear matrix equation if $W_{21} \approx 0$. Unfortunately, it is not easy to see from the present formulation of (3.4) that this singularity is, due to the special structure of the nonlinearities and the right hand side, artificial. Both issues can be more easily addressed after a reformulation of (3.4).

\subsection{Skew-symmetrizing the bottom part. Let}

$$
R=Q+P^{T} \tilde{J} P, \quad \tilde{J}=\left[\begin{array}{cc}
0 & I_{n-k} \\
0 & 0
\end{array}\right]
$$

then $R$ is symmetric if and only if $Q^{T}-Q=P^{T} J P$. The following lemma reveals a particular useful nonlinear matrix equation satisfied by $(P, R)$.

Lemma 4.1. Let $R=Q+P^{T} \tilde{J} P$ be symmetric. Then the matrix pair $(P, Q)$ is a solution of (3.4) if and only if $(P, R)$ is a solution of

$$
\left[\begin{array}{c}
P \\
R
\end{array}\right] W_{11}-\left[\begin{array}{cc}
W_{22} & W_{23} \\
W_{21}^{T} J & W_{11}^{T}
\end{array}\right]\left[\begin{array}{c}
P \\
R
\end{array}\right]+\left[\begin{array}{c}
\Phi_{1}(P, R) \\
\Phi_{2}(P, R)-P^{T} J W_{21}
\end{array}\right]=\left[\begin{array}{l}
W_{21} \\
W_{31}
\end{array}\right]
$$

where

$$
\begin{aligned}
\Phi_{1}(P, R)= & W_{23}\left(P^{T} \tilde{J} P\right)+P\left(J W_{23}\right)^{T} P+P W_{13}\left(R-P^{T} \tilde{J} P\right), \\
\Phi_{2}(P, R)= & \left(R-P^{T} \tilde{J}^{T} P\right) W_{23}^{T} J^{T} P-P^{T} J W_{23}\left(R-P^{T} \tilde{J}^{T} P\right)^{T} \\
& +\left(R-P^{T} \tilde{J} P\right)^{T} W_{13}\left(R-P^{T} \tilde{J} P\right)-P^{T} J W_{22} P .
\end{aligned}
$$

Proof. Adding the top part of (3.4) premultiplied by $P^{T} J$,

$P^{T} J W_{21}=P^{T} J P W_{11}-P^{T} J W_{22} P-P^{T} J W_{23} Q+P^{T} J P\left(J W_{23}\right)^{T} P+P^{T} J P W_{13} Q$,

to the bottom part of (3.4) yields the transformed equation (4.2) after some basic algebraic manipulations.

The reformulated equation (4.2) has the advantage that the nonlinear function $\Phi_{2}(P, R)$, the right hand side term $W_{31}$, as well as the coupling term $-W_{21}^{T} J P-$ $P^{T} J W_{21}$ are skew-symmetric. Hence, these terms belong to the range of the operator $R \mapsto R W_{11}-W_{11}^{T} R$ provided that $W_{11}$ is nonderogatory. This indicates that the singularity caused by this operator is indeed artificial. 
4.2. Solving the decoupled linearized equation. Linearizing (4.2) around $(P, R)=(0,0)$ yields

$$
\tilde{\mathcal{T}}(P, R)=\left[\begin{array}{l}
W_{21} \\
W_{31}
\end{array}\right],
$$

where the operator $\tilde{\mathcal{T}}: \mathbb{R}^{2(n-k) \times k} \times \mathbb{R}^{k \times k} \rightarrow \mathbb{R}^{2(n-k) \times k} \times \mathbb{R}^{k \times k}$ is given by

$$
\tilde{\mathcal{T}}:(P, R) \mapsto\left[\begin{array}{c}
P \\
R
\end{array}\right] W_{11}-\left[\begin{array}{cc}
W_{22} & W_{23} \\
W_{21}^{T} J & W_{11}^{T}
\end{array}\right]\left[\begin{array}{c}
P \\
R
\end{array}\right]-\left[\begin{array}{c}
0 \\
P^{T} J W_{21}
\end{array}\right] .
$$

Note that we sometimes identify $(X, Y) \sim\left[\begin{array}{l}X \\ Y\end{array}\right]$ for notational convenience. It is assumed that the perturbation $E$ is considerably small implying that $W_{21}$ is small. Hence, $W_{21}^{T} J P$ and $P^{T} J W_{21}$ can be regarded as weak coupling terms. Let us neglect these terms and consider the operator

$$
\mathcal{T}:(P, R) \mapsto\left[\begin{array}{c}
P \\
R
\end{array}\right] W_{11}-\left[\begin{array}{cc}
W_{22} & W_{23} \\
0 & W_{11}^{T}
\end{array}\right]\left[\begin{array}{l}
P \\
R
\end{array}\right],
$$

which allows an easy characterization. In the following lemma, $\operatorname{Sym}(k)$ denotes the set of all symmetric $k \times k$ matrices, and Skew $(k)$ the set of all skew-symmetric $k \times k$ matrices.

Lemma 4.2. Consider the operator $\mathcal{T}$ defined by (4.4) with domain and codomain restricted to $\operatorname{dom} \mathcal{T}=\mathbb{R}^{2(n-k) \times k} \times \operatorname{Sym}(k)$ and codom $\mathcal{T}=\mathbb{R}^{2(n-k) \times k} \times \operatorname{Skew}(k)$, respectively. Then $\mathcal{T}$ is onto if and only if $W_{11}$ is nonderogatory and $\lambda\left(W_{11}\right) \cap \lambda\left(W_{22}\right)=$ $\emptyset$.

Proof. If $W_{11}$ is nonderogatory and $\lambda\left(W_{11}\right) \cap \lambda\left(W_{22}\right)=\emptyset$ then we can apply Propositions 2.5 and 2.4 combined with backward substitution to show that $\mathcal{T}$ is onto. For the other direction, assume that $\mathcal{T}$ is onto. The nonderogatority of $W_{11}$ is a consequence of Proposition 2.5; it remains to show that $\lambda\left(W_{11}\right) \cap \lambda\left(W_{22}\right)=\emptyset$. By continuity, we may assume w.l.o.g. that there is a nonsingular matrix $X$ so that $\Lambda=$ $X^{-1} W_{11} X$ is diagonal with diagonal elements $\lambda_{1}, \ldots, \lambda_{k} \in \mathbb{C}$. Then there is a matrix $\tilde{R}_{0} \in \mathbb{C}^{k \times k}$ so that every solution of the transformed equation $\tilde{R} \Lambda-\bar{\Lambda} \tilde{R}=X^{-1} W_{31} X$ has the form

$$
\tilde{R}=\tilde{R}_{0}+\sum_{i=1}^{k} \alpha_{i} e_{i} e_{i}^{T}, \quad \alpha_{1}, \ldots, \alpha_{k} \in \mathbb{C} .
$$

Inserting this representation into the equation $\tilde{P} \Lambda-W_{22} \tilde{P}-W_{23} X^{-T} \tilde{R}=W_{13} X$ leads to the $k$ separate equations

$$
\left[\begin{array}{ll}
\lambda_{i} I-W_{22} & b_{i}
\end{array}\right]\left[\begin{array}{c}
\tilde{p}_{i} \\
\alpha_{i}
\end{array}\right]=\left(W_{13} X+W_{23} \tilde{R}_{0}\right) e_{i},
$$

where $\tilde{p}_{i}$ and $b_{i}$ denote the $i$ th columns of $\tilde{P}$ and $W_{23} X^{-T}$, respectively. Equation (4.5) has a solution for any $W_{13} \in \mathbb{R}^{2(n-k) \times k}$ if and only if $\left[\lambda_{i} I-W_{22}, b_{i}\right]$ has full rank $2(n-k)$. This implies $\lambda_{i} \notin \lambda\left(W_{22}\right)$, since otherwise

$$
\operatorname{rank}\left(\left[\begin{array}{ll}
\lambda_{i} I-W_{22} & b_{i}
\end{array}\right]\right) \leq \operatorname{rank}\left(\lambda_{i} I-W_{22}\right)+1 \leq 2(n-k)-1,
$$

where we used the fact that the geometric multiplicity of each eigenvalue of the skewHamiltonian matrix $W_{22}$ is at least two [4, Thm. 1]. Thus $\lambda\left(W_{11}\right) \cap \lambda\left(W_{22}\right)=\emptyset$, which concludes the proof. 
For the remainder of this section only the restricted operator $\mathcal{T}$ will be considered and it will be assumed that this operator is onto. Note that for $E=0$ the latter is equivalent to the assumption that $\mathcal{X}$ is semi-simple, see Definition 2.3. The dimensions of the matrix spaces $\operatorname{Skew}(k)$ and $\operatorname{Sym}(k)$ differ by $k$. More precisely, it can be shown that the set of solutions corresponding to a particular right hand side in the codomain of $\mathcal{T}$ form an affine subspace of dimension $k$ [4]. In view of an earlier remark one should pick a solution that has minimal norm. Using the Frobenius norm this solution is uniquely determined as the following lemma shows.

Lemma 4.3. Let $\mathcal{T}$ be defined as in (4.4) and let $\left(W_{21}, W_{31}\right) \in$ codom $\mathcal{T}$. Then there is one and only one matrix pair $\left(P_{\star}, R_{\star}\right) \in \operatorname{dom} \mathcal{T}$ satisfying

$$
\left\|\left(P_{\star}, R_{\star}\right)\right\|_{F}=\min _{(P, R) \in \operatorname{dom} \mathcal{T}}\left\{\|(P, R)\|_{F} \mid \mathcal{T}(P, R)=\left[\begin{array}{c}
W_{21} \\
W_{31}
\end{array}\right]\right\} .
$$

Proof. Using the second part of Proposition 2.5 the constraint $(P, R) \in \operatorname{dom} \mathcal{T}$ in (4.6) can be dropped. Let us define

$$
K_{\mathcal{T}}:=W_{11}^{T} \otimes I-I \otimes\left[\begin{array}{cc}
W_{22} & W_{23} \\
0 & W_{11}^{T}
\end{array}\right],
$$

where ' $\otimes$ ' denotes the Kronecker product of two matrices [5, Sec. 4.5.5]. Using the vec operator, which stacks the columns of a matrix into one long vector, the minimization problem (4.6) can be written in the form

$$
\min _{x \in \mathbb{R}^{(2 n-k) \times k}}\left\{\|x\|_{2}: K_{\mathcal{T}} \cdot x=w\right\},
$$

where $w=\operatorname{vec}\left(\left[\begin{array}{l}W_{21} \\ W_{31}\end{array}\right]\right)$. Well-known results about linear least-squares problems show that (4.7) has a unique minimum given by $K_{\mathcal{T}}^{\dagger} \cdot w$, where $K_{\mathcal{T}}^{\dagger}$ denotes the pseudoinverse of $K_{\mathcal{T}}$ [5, Sec. 5.5.4].

This lemma allows us to define an operator

$$
\mathcal{T}^{\dagger}: \operatorname{codom} \mathcal{T} \rightarrow \operatorname{dom} \mathcal{T}
$$

which maps a matrix pair $\left(W_{21}, W_{31}\right)$ to the solution of (4.6). A sensible choice of norm for $\mathcal{T}^{\dagger}$ is the one induced by the Frobenius norm,

$$
\left\|\mathcal{T}^{\dagger}\right\|:=\sup _{\substack{\left\|\left(W_{21}, W_{31}\right)\right\|_{F}=1 \\\left(W_{21}, W_{31}\right) \in \operatorname{codom} \mathcal{T}}}\left\|\mathcal{T}^{\dagger}\left(W_{21}, W_{31}\right)\right\|_{F} .
$$

4.3. Solving the coupled linearized equation. The key to solving the coupled equation (4.3) is to note that $\tilde{\mathcal{T}}$ can be decomposed into $\mathcal{T}-\triangle \mathcal{T}_{W}$, where $\triangle \mathcal{T}: \operatorname{dom} \mathcal{T} \rightarrow$ codom $\mathcal{T}$ is defined by

$$
\triangle \mathcal{T}:(P, R) \mapsto\left[\begin{array}{c}
0 \\
P^{T} J W_{21}+W_{21}^{T} J P
\end{array}\right] .
$$

This implies that the composed operator $\mathcal{T}^{\dagger} \circ \Delta \mathcal{T}: \operatorname{dom} \mathcal{T} \rightarrow$ dom $\mathcal{T}$ is well-defined, its norm is again the one induced by the Frobenius norm.

Lemma 4.4. If $\mathcal{T}$ is onto and $\delta:=\left\|\mathcal{T}^{\dagger} \circ \triangle \mathcal{T}\right\|<1$ then

$$
\tilde{\mathcal{T}}^{\tilde{\dagger}}\left(W_{21}, W_{31}\right):=\sum_{i=0}^{\infty}\left(\mathcal{T}^{\dagger} \circ \triangle \mathcal{T}\right)^{i} \circ \mathcal{T}^{\dagger}\left(W_{21}, W_{31}\right)
$$


is a solution of (4.3).

Proof. If $\delta<1$ then

$$
\left\|\sum_{i=0}^{\infty}\left(\mathcal{T}^{\dagger} \circ \triangle \mathcal{T}\right)^{i} \circ \mathcal{T}^{\dagger}\right\| \leq \sum_{i=0}^{\infty} \delta^{i}\left\|\mathcal{T}^{\dagger}\right\|=\frac{\left\|\mathcal{T}^{\dagger}\right\|}{1-\delta}
$$

implying that the infinite sum in (4.10) converges absolutely. Moreover, pre-multiplying (4.10) with $\mathcal{T}-\triangle \mathcal{T}$ shows that $\tilde{\mathcal{T}}^{\tilde{\dagger}}\left(W_{21}, W_{31}\right)$ solves (4.3).

Inequality (4.11) yields the bound

$$
\left\|\tilde{\mathcal{T}}^{\tilde{\dagger}}\left(W_{21}, W_{31}\right)\right\|_{F} \leq \frac{\left\|\mathcal{T}^{\dagger}\right\|}{1-\delta} \cdot\left\|\left(W_{21}, W_{31}\right)\right\|_{F} .
$$

An upper bound for the quantity $\delta$ is clearly given by $2\left\|\mathcal{T}^{\dagger}\right\|\left\|W_{21}\right\|_{F}$. It should be stressed that $\tilde{\mathcal{T}}^{\tilde{\dagger}}: \operatorname{codom} \mathcal{T} \rightarrow \operatorname{dom} \mathcal{T}$ does not necessarily give the solution of smallest norm. However, if $\|\triangle \mathcal{T}\|$ is sufficiently small it can be expected to be rather close to it.

LEMma 4.5. Under the assumption of Lemma 4.4 let $\tilde{\mathcal{T}}^{\dagger}: \operatorname{codom} \mathcal{T} \rightarrow \operatorname{dom} \mathcal{T}$ denote the operator that maps a pair $\left(W_{21}, W_{31}\right)$ to the minimal norm solution of the coupled equation (4.3). Then

$$
\lim _{\Delta \mathcal{T} \rightarrow 0} \tilde{\mathcal{T}}^{\tilde{\dagger}}=\lim _{\triangle \mathcal{T} \rightarrow 0} \tilde{\mathcal{T}}^{\dagger}=\mathcal{T}^{\dagger} .
$$

Proof. Lemma 4.4 shows that the coupled equation (4.3) has, for a given right hand side in codom $\mathcal{T}$, a nonempty set of solutions. This set is, according to Proposition 2.5, a subset of dom $\mathcal{T}$. The solution of minimal norm is uniquely defined, for reasons similar to those that have been used in the proof of Lemma 4.3. Hence, the operator $\tilde{\mathcal{T}}^{\dagger}$ is well-defined. By checking the four Penrose conditions it can be shown that $\tilde{\mathcal{T}}^{\tilde{\dagger}}=\left(\mathcal{T}-\triangle \mathcal{T} \circ\left(\mathcal{T}^{\dagger} \circ \mathcal{T}\right)\right)^{\dagger}$. Equalities (4.13) follow from the fact that the ranges of $\mathcal{T}-\triangle \mathcal{T} \circ\left(\mathcal{T}^{\dagger} \circ \mathcal{T}\right), \tilde{\mathcal{T}}$ and $\mathcal{T}$ have equal dimensions [16, Sec. III.3].

We remark that Lemma 4.4 and Lemma 4.5 are not restricted to perturbations of the form (4.9). In fact, they hold for any $\triangle \mathcal{T}: \operatorname{dom} \mathcal{T} \rightarrow$ codom $\mathcal{T}$ satisfying $\left\|\mathcal{T}^{\dagger} \circ \triangle \mathcal{T}\right\|<1$.

4.4. Solving the nonlinear equation. Using the terminology developed above we can rewrite the nonlinear equation (4.2) in the more convenient form

$$
\tilde{\mathcal{T}}(P, R)+\Phi(P, R)=\left[\begin{array}{l}
W_{21} \\
W_{31}
\end{array}\right],
$$

where $\Phi(P, R)=\left[\Phi_{1}(P, R)^{T}, \Phi_{2}(P, R)^{T}\right]^{T}$.

THEOREM 4.6. Let the matrices $W_{i j}$ be defined by (3.1) and assume that the operator $\mathcal{T}$ defined by (4.4) is onto in the sense of Lemma 4.2. Assume that $\delta=$ $2\left\|\mathcal{T}^{\dagger}\right\|\left\|W_{21}\right\|_{F}<1$, where $\left\|\mathcal{T}^{\dagger}\right\|$ is defined by (4.8). Set

$$
\gamma=\left\|\left(W_{21}, W_{31}\right)\right\|_{F}, \quad \eta=\left\|\left[\begin{array}{cc}
W_{23}^{T} J^{T} & W_{13} \\
W_{22} & W_{23}
\end{array}\right]\right\|_{F}, \quad \kappa=\frac{\left\|\mathcal{T}^{\dagger}\right\|}{1-\delta} .
$$

Then if

$$
8 \gamma \kappa<1, \quad 20 \gamma \eta \kappa^{2}<1
$$


there is a solution $(P, R)$ of (4.14) satisfying

$$
\|(P, R)\|_{F} \leq 2 \gamma \kappa .
$$

Proof. We adapt the technique used by Stewart [15, Sec. 3] and solve (4.14) by constructing an iteration. First, some facts about the nonlinearities are required:

$$
\begin{aligned}
& \left\|\Phi_{1}(P, R)\right\|_{F} \leq\left\|W_{13}\right\|_{F}\left(\|P\|_{F}\|R\|_{F}+\|P\|_{F}^{3}\right)+2\left\|W_{23}\right\|_{F}\|P\|_{F}^{2} \\
& \left\|\Phi_{2}(P, R)\right\|_{F} \leq \eta\|(P, R)\|_{F}^{2}+\left\|W_{13}\right\|_{F}\left(2\|P\|_{F}^{2}\|R\|_{F}+\|P\|_{F}^{4}\right)+2\left\|W_{23}\right\|_{F}\|P\|_{F}^{3} \\
\Rightarrow & \|\Phi(P, R)\|_{F} \leq(1+\sqrt{3}) \eta\|(P, R)\|_{F}^{2}+(\sqrt{2}+\sqrt{3}) \eta\|(P, R)\|_{F}^{3}+\eta\|(P, R)\|_{F}^{4} .
\end{aligned}
$$

Using a rough estimate, we have $\|\Phi(P, R)\|_{F} \leq 4 \eta\|(P, R)\|_{F}^{2}$ for $\|(P, R)\|_{F} \leq 1 / 4$. Similarly, it can be shown that

$$
\begin{aligned}
\|\Phi(\hat{P}, \hat{R})-\Phi(P, R)\|_{F} \leq & {\left[2(1+\sqrt{3}) \eta \max \left\{\|(\hat{P}, \hat{R})\|_{F},\|(P, R)\|_{F}\right\}\right.} \\
& +4(\sqrt{2}+\sqrt{3}) \eta \max \left\{\|(\hat{P}, \hat{R})\|_{F},\|(P, R)\|_{F}\right\}^{2} \\
& \left.+8 \eta \max \left\{\|(\hat{P}, \hat{R})\|_{F},\|(P, R)\|_{F}\right\}^{3}\right] \cdot\|(\hat{P}-P, \hat{R}-R)\|_{F} \\
\leq & 10 \eta \max \left\{\|(\hat{P}, \hat{R})\|_{F},\|(P, R)\|_{F}\right\} \cdot\|(\hat{P}-P, \hat{R}-R)\|_{F},
\end{aligned}
$$

where the latter inequality holds for $\max \left\{\|(P, R)\|_{F},\|(\hat{P}, \hat{R})\|_{F}\right\} \leq 1 / 4$. Next, we define a sequence by $\left(P_{0}, R_{0}\right)=(0,0)$ and

$$
\left(P_{k+1}, R_{k+1}\right)=\tilde{\mathcal{T}}^{\tilde{\dagger}}\left(W_{21}, W_{31}\right)+\tilde{\mathcal{T}}^{\tilde{\dagger}} \circ \Phi\left(P_{k}, R_{k}\right) .
$$

Note that this iteration is well-defined as $\Phi: \operatorname{dom} \mathcal{T} \rightarrow \operatorname{codom} \mathcal{T}$. We show by induction that the iterates stay bounded. Under the assumption $\left\|\left(P_{k}, R_{k}\right)\right\|<2 \gamma \kappa \leq$ $1 / 4$ it follows that

$$
\left\|\left(P_{k+1}, R_{k+1}\right)\right\|_{F} \leq \kappa\left(\gamma+4 \eta\left\|\left(P_{k}, R_{k}\right)\right\|_{F}^{2}\right)<2 \gamma \kappa .
$$

The operator $\tilde{\mathcal{T}}^{\tilde{\dagger}} \Phi$ is a contraction on $\mathcal{D}=\left\{(P, R):\|(P, R)\|_{F}<2 \gamma \kappa\right\}$ since

$$
\left\|\tilde{\mathcal{T}}^{\tilde{\dagger}} \circ \Phi(\hat{P}, \hat{R})-\tilde{\mathcal{T}}^{\tilde{\dagger}} \circ \Phi(P, R)\right\|_{F} \leq 20 \gamma \eta \kappa^{2}\|(\hat{P}-P, \hat{R}-R)\|_{F}<\|(\hat{P}-P, \hat{R}-R)\|_{F}
$$

for all $(P, R) \in \mathcal{D}$ and $(\hat{P}, \hat{R}) \in \mathcal{D}$. Thus, the contraction mapping theorem [12] shows that the sequence $\left(P_{k}, R_{k}\right)$ converges to a fixed point, which solves (4.14).

COROLlary 4.7. Under the assumptions of Theorem 4.6 there is a solution $(P, Q)$ of the quadratic matrix equation (3.4) satisfying $Q^{T}-Q=P^{T} J P$ and

$$
\|(P, Q)\|_{F} \leq 2 \gamma \kappa+4 \gamma^{2} \kappa^{2}<2.5 \gamma \kappa .
$$

Proof. The result is a direct consequence of the relationship $Q=R-P^{T} \tilde{J} P$.

5. Perturbations bounds and a condition number. From the discussion in Section 3 it follows that Corollary 4.7 yields the existence of an isotropic invariant subspace $\hat{\mathcal{X}}$ of $W+E$ close to $\mathcal{X}$, which is an isotropic invariant subspace of the unperturbed matrix $W$.

COROLlary 5.1. Under the assumptions of Theorem 4.6 there is an isotropic invariant subspace $\hat{\mathcal{X}}$ of the skew-Hamiltonian matrix $W+E$ so that

$$
\sqrt{\tan ^{2} \theta_{1}(\mathcal{X}, \hat{\mathcal{X}})+\cdots+\tan ^{2} \theta_{k}(\mathcal{X}, \hat{\mathcal{X}})} \leq 2 \gamma \kappa+4 \gamma^{2} \kappa^{2}<2.5 \gamma \kappa
$$


where $\theta_{i}(\mathcal{X}, \hat{\mathcal{X}}), i=1, \ldots, k$, are the canonical angles between $\mathcal{X}$ and $\hat{\mathcal{X}}$.

Proof. Inequality (5.1) follows from Corollary 4.7 using the fact that $\tan \theta_{i}(\mathcal{X}, \hat{\mathcal{X}})$, $i=1, \ldots, k$, are the singular values of the matrix $\left[P^{T}, Q^{T}\right]^{T}$.

The catch of this corollary is that it works with quantities that are usually not known. For example, the operator $\mathcal{T}$, used to define $\kappa$, explicitely depends on the matrix $W+E$. However, often not the perturbation $E$ itself but only an upper bound on its norm is given. For this reason, given a partitioning (2.3) let us use the unperturbed data to define an operator $\mathcal{T}_{W}: \operatorname{dom} \mathcal{T} \rightarrow \operatorname{codom} \mathcal{T}$ as follows:

$$
\mathcal{T}_{W}:(P, Q) \mapsto\left[\begin{array}{c}
P \\
Q
\end{array}\right] A_{11}-\left[\begin{array}{c}
P \\
Q
\end{array}\right]\left[\begin{array}{ccc}
A_{22} & G_{22} & -G_{12}^{T} \\
H_{22} & A_{22}^{T} & A_{12}^{T} \\
0 & 0 & A_{11}^{T}
\end{array}\right]
$$

The operator $\mathcal{T}_{W}^{\dagger}$ and its norm are defined in the same sense as $\mathcal{T}^{\dagger}$ and $\left\|\mathcal{T}^{\dagger}\right\|$.

TheOREm 5.2. Let $U=\left[X, Z, J^{T} X, J^{T} Z\right]$ be orthogonal symplectic and suppose that $\mathcal{X}=\operatorname{span} X$ is a semi-simple isotropic invariant subspace of the skewHamiltonian matrix $W$ so that

$$
U^{T} W U=\left[\begin{array}{cccc}
A_{11} & A_{12} & G_{11} & G_{12} \\
0 & A_{22} & -G_{12}^{T} & G_{22} \\
0 & 0 & A_{11}^{T} & 0 \\
0 & H_{22} & A_{12}^{T} & A_{22}^{T}
\end{array}\right]
$$

Given a skew-Hamiltonian perturbation E, let

$$
U^{T} E U=\left[\begin{array}{cccc}
E_{11} & E_{12} & E_{13} & E_{14} \\
E_{21} & E_{22} & -E_{14}^{T} & E_{24} \\
E_{31} & E_{32} & E_{11}^{T} & E_{21}^{T} \\
-E_{32}^{T} & E_{42} & E_{12}^{T} & E_{22}^{T}
\end{array}\right]
$$

Assume that $\hat{\delta}=\sqrt{3}\left\|\mathcal{T}_{W}^{\dagger}\right\| \cdot\|E\|_{F}<1$, where $\mathcal{T}_{W}^{\dagger}$ is defined by (5.2). Set

$$
\hat{\gamma}=\left\|\left[\begin{array}{c}
E_{21} \\
E_{31} \\
E_{32}^{T}
\end{array}\right]\right\|_{F}, \hat{\eta}=\left\|\left[\begin{array}{ccc}
A_{12} & G_{11} & G_{12} \\
A_{22} & -G_{12}^{T} & G_{22} \\
H_{22} & A_{12}^{T} & A_{22}^{T}
\end{array}\right]\right\|_{F}+\left\|\left[\begin{array}{ccc}
E_{12} & E_{13} & E_{14} \\
E_{22} & -E_{14}^{T} & E_{24} \\
E_{42} & E_{12}^{T} & E_{22}^{T}
\end{array}\right]\right\|_{F}
$$

and $\hat{\kappa}=\left\|\mathcal{T}_{W}^{\dagger}\right\| /(1-\hat{\delta})$. Then if

$$
8 \hat{\gamma} \hat{\kappa}<1, \quad 20 \hat{\gamma} \hat{\eta} \hat{\kappa}^{2}<1,
$$

there are matrices $P$ and $Q$ satisfying

$$
\|(P, Q)\|_{F} \leq 2 \hat{\gamma} \hat{\kappa}+4 \hat{\gamma}^{2} \hat{\kappa}^{2}<2.5 \hat{\gamma} \hat{\kappa}
$$

so that the columns of

$$
\hat{X}=\left(X+\left[Z, J^{T} Z, J^{T} X\right]\left[\begin{array}{l}
P \\
Q
\end{array}\right]\right)\left(I+P^{T} P+Q^{T} Q\right)^{-1 / 2}
$$

form an orthonormal basis for an isotropic invariant subspace of $\hat{W}=W+E$. 
Proof. First, note that the semi-simplicity of $\mathcal{X}$ implies that $\mathcal{T}_{W}$ is onto. The operator $\tilde{\mathcal{T}}$, defined in Section 4.2, is decomposed into $\mathcal{T}_{W}-\triangle \mathcal{T}_{W}$, where $\triangle \mathcal{T}_{W}$ : $\operatorname{dom} \mathcal{T} \rightarrow \operatorname{codom} \mathcal{T}$ is given by

$$
\triangle \mathcal{T}_{W}:\left[\begin{array}{c}
P \\
R
\end{array}\right] \mapsto\left[\begin{array}{c}
P \\
R
\end{array}\right] E_{11}-\left[\begin{array}{ccc}
E_{22} & E_{24} & -E_{14}^{T} \\
E_{42} & E_{22}^{T} & E_{12}^{T} \\
-E_{32} & -E_{21}^{T} & E_{11}^{T}
\end{array}\right]\left[\begin{array}{c}
P \\
R
\end{array}\right]-\left[\begin{array}{c}
0 \\
F
\end{array}\right]
$$

with $F=P^{T}\left[\begin{array}{l}E_{32}^{T} \\ E_{21}\end{array}\right]$. Hence, $\left\|\triangle \mathcal{T}_{W}\right\| \leq \sqrt{3}\|E\|_{F}$ and Lemma 4.4 implies that

$$
\tilde{\mathcal{T}}^{\tilde{\dagger}}=\sum_{i=0}^{\infty}\left(\mathcal{T}_{W}^{\dagger} \circ \triangle \mathcal{T}_{W}\right)^{i} \circ \mathcal{T}_{W}^{\dagger}
$$

converges absolutely and satisfies $\left\|\tilde{\mathcal{T}}^{\tilde{\dagger}}\right\| \leq \hat{\kappa}$. The remainder of the proof is analogous to the proof of Theorem 4.6.

The bound (5.1) on the canonical angles between $\mathcal{X}$ and $\hat{\mathcal{X}}$ holds with the quantities $\gamma$ and $\kappa$ replaced by $\hat{\gamma}$ and $\hat{\kappa}$ :

$$
\|\tan \Theta(\mathcal{X}, \hat{\mathcal{X}})\|_{F} \leq 2 \hat{\gamma} \hat{\kappa}+4 \hat{\gamma}^{2} \hat{\kappa}^{2}<2.5 \hat{\gamma} \hat{\kappa} .
$$

Similar to the standard notion of the condition number for an invariant subspace of a general matrix [16], we define the structured condition number $c_{W}(\mathcal{X})$ for a semisimple isotropic invariant subspace $\mathcal{X}$ of a skew-Hamiltonian matrix by the quantity that is approximated by $\|\Theta(\mathcal{X}, \hat{\mathcal{X}})\|_{F} / \varepsilon$ as the perturbation level $\varepsilon$ tends to zero. From the bound (5.4) and the expansion of $\tan (\cdot)$ around zero we conclude that $c_{W}(\mathcal{X})$ satisfies

$$
c_{W}(\mathcal{X}):=\lim _{\varepsilon \rightarrow 0} \sup _{\substack{\|E\|_{F} \leq \varepsilon \\ \text { skew-Hamiltonian }}} \frac{\|\Theta(\mathcal{X}, \hat{\mathcal{X}})\|_{F}}{\varepsilon} \leq \alpha\left\|\mathcal{T}_{W}^{\dagger}\right\|
$$

for some $\alpha \leq 2$. The presence of the factor $\alpha$ in this bound is artificial; a slight modification of the proof of Theorem 4.6 shows that $\alpha$ can be made arbitrarily close to one under the assumption that the perturbation $E$ is sufficiently small. This reveals that $\left\|\mathcal{T}_{W}^{\dagger}\right\|$ is an upper bound on $c_{W}(\mathcal{X})$.

To show that $c_{W}(\mathcal{X})$ and $\left\|\mathcal{T}_{W}^{\dagger}\right\|$ actually coincide we construct skew-Hamiltonian perturbations $E$ so that

$$
\lim _{\|E\|_{F} \rightarrow 0}\|\Theta(\mathcal{X}, \hat{\mathcal{X}})\|_{F} /\|E\|_{F} \geq\left\|\mathcal{T}_{W}^{\dagger}\right\| .
$$

holds. Given a block Schur decomposition of the form (5.3), choose matrices $E_{21}$ and $E_{31}$ so that $\left\|\left(E_{21}, E_{31}\right)\right\|_{F}=1,\left\|\mathcal{T}_{W}^{\dagger}\left(E_{21}, E_{31}\right)\right\|_{F}=\left\|\mathcal{T}_{W}^{\dagger}\right\|$, and consider the perturbation

$$
E=\varepsilon \cdot\left[Z, J^{T} X, J^{T} Z\right]\left[\begin{array}{c}
E_{21} \\
E_{31}
\end{array}\right] X^{T} .
$$

By choosing $\varepsilon$ sufficiently small, we may assume that there is an invariant subspace $\hat{\mathcal{X}}$ of $W+E$ satisfying $\|\Theta(\mathcal{X}, \hat{\mathcal{X}})\|_{2}<\frac{\pi}{2}$. This implies the existence of matrices $P$ and $Q$ so that the columns of

$$
\hat{X}=\left(X+\left[Z, J^{T} Z, J^{T} X\right]\left[\begin{array}{l}
P \\
Q
\end{array}\right]\right)\left(I+P^{T} P+Q^{T} Q\right)^{-1 / 2}
$$


form an orthonormal basis of $\hat{\mathcal{X}}$. We have seen that any such matrix pair $(P, Q)$ must satisfy the nonlinear matrix equation

$$
\mathcal{T}_{W}(P, R)-\triangle \mathcal{T}_{W}(P, R)+\Phi(P, R)=\varepsilon\left[\begin{array}{c}
E_{21} \\
E_{31}
\end{array}\right],
$$

where $R, \triangle \mathcal{T}_{W}$ and $\Phi$ are defined as in (4.1), (4.9) and (4.14), respectively. If we decompose

$$
(P, R)=\left(P_{1}+P_{2}, R_{1}+R_{2}\right), \quad\left(P_{1}, R_{1}\right) \in \operatorname{kernel}\left(\mathcal{T}_{W}\right),\left(P_{2}, R_{2}\right) \in \operatorname{kernel}\left(\mathcal{T}_{W}\right)^{\perp},
$$

then

$$
\left(P_{2}, R_{2}\right)=\varepsilon \cdot \mathcal{T}_{W}^{\dagger}\left(E_{21}, E_{31}\right)+\mathcal{T}_{W}^{\dagger} \circ\left[\triangle \mathcal{T}_{W}(P, R)-\Phi(P, R)\right] .
$$

Since $\|(P, R)\|=\mathcal{O}(\varepsilon)$, it follows that $\left\|\triangle \mathcal{T}_{W}(P, R)-\Phi(P, R)\right\|_{F}=\mathcal{O}\left(\varepsilon^{2}\right)$ and thus

$$
\lim _{\varepsilon \rightarrow 0}\left\|\left(P_{2}, R_{2}\right)\right\|_{F} / \varepsilon=\left\|\mathcal{T}_{W}^{\dagger}\left(E_{21}, E_{31}\right)\right\|_{F}=\left\|\mathcal{T}_{W}^{\dagger}\right\|
$$

Combining this equality with $\|(P, R)\|_{F} \geq\left\|\left(P_{2}, R_{2}\right)\right\|_{F}$ and $\|\Theta(\mathcal{X}, \hat{\mathcal{X}})\|_{F}=\|(P, R)\|_{F}+$ $\mathcal{O}\left(\varepsilon^{2}\right)$ yields the desired result:

$$
\lim _{\varepsilon \rightarrow 0}\|\Theta(\mathcal{X}, \hat{\mathcal{X}})\|_{F} / \varepsilon \geq\left\|\mathcal{T}_{W}^{\dagger}\right\|
$$

6. On the computation of $\left\|\mathcal{T}_{W}^{\dagger}\right\|$. The discussion above shows that $\left\|\mathcal{T}_{W}^{\dagger}\right\|$ measures the sensitivity of an isotropic invariant subspace. It remains to compute this quantity. It turns out that $\left\|\mathcal{T}_{W}^{\dagger}\right\|$ is considerably easy to compute if $k=1$ (real eigenvectors).

Lemma 6.1. Let $\lambda \in \mathbb{R}$ be an eigenvalue of the skew-Hamiltonian matrix $W$ with algebraic multiplicity two, and let $x$ be an associated eigenvector satisfying $\|x\|_{2}=1$. Given a partitioning of the form (5.3) with respect to $x$ it follows that

$$
\left\|\mathcal{T}_{W}^{\dagger}\right\|=\sigma_{\min }\left(W_{\lambda}\right)^{-1},
$$

where $\sigma_{\min }$ denotes the minimum singular value of a matrix and

$$
W_{\lambda}=\left[\begin{array}{ccc}
A_{22}-\lambda I & G_{22} & -G_{12}^{T} \\
H_{22} & A_{22}^{T}-\lambda I & A_{12}^{T}
\end{array}\right] .
$$

Proof. The operator $\mathcal{T}_{W}$ can be identified with $\left[\begin{array}{c}W_{\lambda} \\ 0\end{array}\right]$. Hence,

$$
\left\|\mathcal{T}_{W}^{\dagger}\right\|=\sup _{\|x\|_{2}=1}\left\|\mathcal{T}_{W}^{\dagger}(x, 0)\right\|_{2}=\sup _{\|x\|_{2}=1}\left\|W_{\lambda}^{\dagger} x\right\|_{2}=\sigma_{\min }\left(W_{\lambda}\right)^{-1}
$$

using the fact that the space of $1 \times 1$ skew-symmetric matrices is $\{0\}$.

If $U^{T} W U$ is in skew-Hamiltonian Schur form (2.2) then $H_{22}=0$ and $A_{22}$ is in real Schur form. Then the computation of $\left\|\mathcal{T}_{W}^{\dagger}\right\|$ becomes particularly cheap. Construct an orthogonal matrix $Q$ so that

$$
W_{\lambda} Q=\left[\begin{array}{ccc}
T_{11} & T_{12} & 0 \\
0 & T_{22}^{T} & 0
\end{array}\right]
$$


with upper triangular matrices $T_{11}$ and $T_{22}$. Since $Q$ can be represented as a product of $\mathcal{O}(n)$ Givens rotations, see [5, Sec. 12.5], the computation of $T_{11}, T_{12}$ and $T_{22}$ requires $\mathcal{O}\left(n^{2}\right)$ floating point operations (flops). In this case, one of the condition number estimators for triangular matrices $[6, \mathrm{Ch} .15]$ can be used to estimate

$$
\left\|\left[\begin{array}{cc}
T_{11} & T_{12} \\
0 & T_{22}^{T}
\end{array}\right]^{-1}\right\|_{2}=\sigma_{\min }\left(W_{\lambda} U\right)^{-1}=\sigma_{\min }\left(W_{\lambda}\right)^{-1}
$$

within $\mathcal{O}\left(n^{2}\right)$ flops.

The case $k>1$ is more complicated. A possible but quite expensive option is provided by the Kronecker product approach that was already used in the proof of Lemma 4.3. Let

$$
K_{\mathcal{T}_{W}}:=A_{11}^{T} \otimes I-I \otimes\left[\begin{array}{ccc}
A_{22} & G_{22} & -G_{12}^{T} \\
H_{22} & A_{22}^{T} & A_{12}^{T} \\
0 & 0 & A_{11}^{T}
\end{array}\right]
$$

and let the columns of $K_{\text {Skew }}$ form an orthonormal basis for all vectors in vec(codom $\mathcal{T}$ ). Then $\left\|\mathcal{T}_{W}^{\dagger}\right\|$ is given by the minimum singular value of the matrix $K_{\text {Skew }}^{T} K_{\mathcal{T}_{W}}$. Note that this is an $(2 n k-k(3 k+1) / 2) \times\left(2 n k-k^{2}\right)$ matrix and thus a direct method for computing its minimum singular value requires $\mathcal{O}\left(k^{3} n^{3}\right)$ flops.

Another approach would consist of adapting a condition estimator for Sylvester equations $[3,9]$ to estimate $\left\|\mathcal{T}_{W}^{\dagger}\right\|$. This would require the application of $\mathcal{T}_{W}^{\dagger}$ (and its dual) to particular elements of $\operatorname{codom} \mathcal{T}$ (and $\operatorname{dom} \mathcal{T}$ ). The efficient and reliable computation of these "matrix-vector products" is a delicate task, see e.g. [7], and beyond the scope of this paper.

7. Numerical Example. Algorithms for computing the derived condition numbers for eigenvectors of skew-Hamiltonian matrices have been implemented in Fortran 77. They are part of HAPACK [1], a prospective software library for solving eigenvalue problems with Hamiltonian, skew-Hamiltonian or block cyclic structures. Let us illustrate their use with the following $2 n \times 2 n$ skew-Hamiltonian matrix:

$$
W_{n}=\left[\begin{array}{cccc|cccc}
0 & -1 & \cdots & -1 & 0 & 1 & \cdots & 1 \\
0 & -1 & \cdots & -1 & -1 & 0 & \ddots & \vdots \\
\vdots & \ddots & \ddots & \vdots & \vdots & \ddots & \ddots & 1 \\
0 & \cdots & 0 & -1 & -1 & \cdots & -1 & 0 \\
\hline 0 & \cdots & \cdots & 0 & 0 & 0 & \cdots & 0 \\
\vdots & & & \vdots & -1 & -1 & \ddots & \vdots \\
\vdots & & & \vdots & \vdots & \vdots & \ddots & 0 \\
0 & \cdots & \cdots & 0 & -1 & -1 & \cdots & -1
\end{array}\right] .
$$

We computed exact values of $\left\|\mathcal{T}_{W}^{\dagger}\right\|$ for the eigenvector $e_{1}$ of $W_{n}, n=2, \ldots, 30$. Furthermore, we applied the algorithm proposed in Section 6 to produce estimates of $\left\|\mathcal{T}_{W}^{\dagger}\right\|$. These theoretical results were compared with practical observations in the following way. A skew-Hamiltonian matrix $E$ with random entries chosen from $N(0,1)$ had been scaled so that $\|E\|_{F}=10^{-10}$. Using HAPACK routines, we computed eigenvectors $v$ and $w$ corresponding to two identical eigenvalues of $W_{n}+E$ that have smallest absolute value. Let the columns of $U$ form an orthonormal basis for 


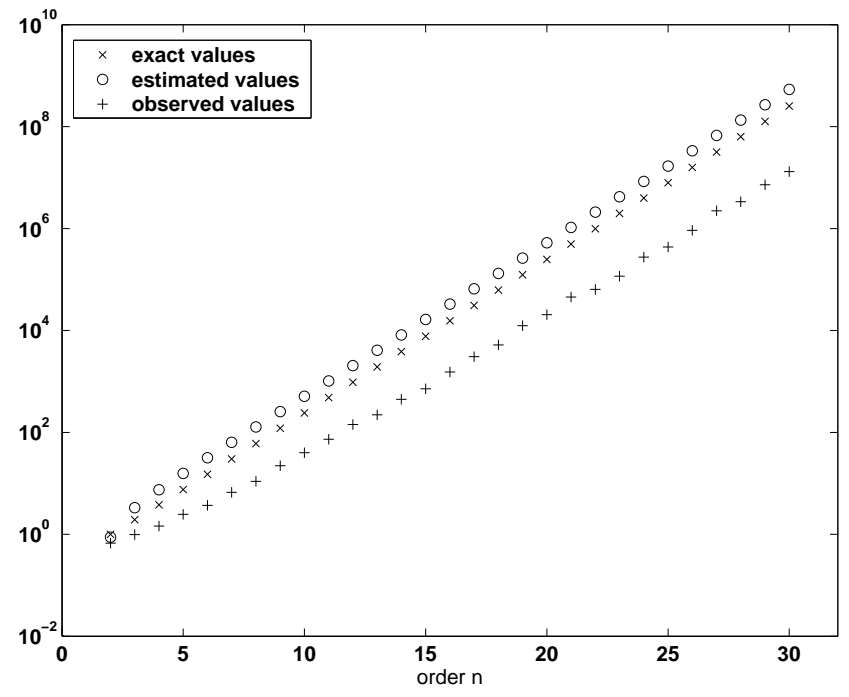

FIG. 7.1. Exact, estimated and observed values of $\left\|\mathcal{T}_{W}^{\dagger}\right\|$ for the eigenvector $e_{1}$ of $W_{n}$.

$\operatorname{span}\{v, w\}^{\perp}$. Then the sine of the angle between $\operatorname{span}\left\{e_{1}\right\}$ and $\operatorname{span}\{v, w\}$ is given by $\left\|U^{H} e_{1}\right\|_{2}$. The observed value of $\left\|\mathcal{T}_{W}^{\dagger}\right\|$ was taken as the maximum over all quantities $10^{10} \cdot\left\|U^{H} e_{1}\right\|_{2}$ for 500 different samples of $E$. The results of the computations, which were performed in a Compaq Visual Fortran environment, are displayed in Figure 7.1. It turns out that the exact value of $\left\|\mathcal{T}_{W}^{\dagger}\right\|$ is underestimated for $n=2$ by a factor of 0.88 and overestimated for all other values of $n$ by a factor of at most 2.2. Furthermore, the exact value is consistently larger than the observed value, by a factor of at most 20 .

8. Conclusions. While the change of eigenvalues under structured perturbations has received a lot of attraction, for a recent work in this area see e.g. [17], invariant subspaces have been much less studied. An extensive perturbation analysis for (block) Hamiltonian Schur forms has been presented in [10]. However, we are not aware of any work on perturbation theory for eigenvectors or invariant subspaces of skew-Hamiltonian matrices. Therefore, we believe that our results are novel. The obtained condition numbers reflect the actual sensitivity of isotropic invariant subspaces rather well, at least for the numerical example presented in the previous section. We hope that the integration of these condition numbers in HAPACK [1] will show whether their usefulness stands the test of practical applications.

9. Acknowledgments. The author thanks Dr. Michael Karow, Prof. Volker Mehrmann and Prof. Ji-guang Sun for useful discussions. This work was carried out while the author was at CESAME, Université catholique de Louvain. The hospitality of this institute is gratefully acknowledged.

\section{REFERENCES}

[1] P. Benner and D. Kressner. Fortran 77 subroutines for computing the eigenvalues of Hamiltonian matrices II. In preparation. See also http://www.math.tu-berlin.de/ ${ }^{k r e s s n e r / ~}$ hapack/, 2004. 
[2] A. Bunse-Gerstner. Matrix factorizations for symplectic $Q R$-like methods. Linear Algebra Appl., 83:49-77, 1986.

[3] R. Byers. A LINPACK-style condition estimator for the equation $A X-X B^{T}=C$. IEEE Trans. Automat. Control, 29(10):926-928, 1984.

[4] H. Faßbender, D. S. Mackey, N. Mackey, and H. Xu. Hamiltonian square roots of skewHamiltonian matrices. Linear Algebra Appl., 287(1-3):125-159, 1999.

[5] G. H. Golub and C. F. Van Loan. Matrix Computations. Johns Hopkins University Press, Baltimore, MD, third edition, 1996.

[6] N. J. Higham. Accuracy and stability of numerical algorithms. Society for Industrial and Applied Mathematics (SIAM), Philadelphia, PA, second edition, 2002.

[7] A. S. Hodel and P. Misra. Least-squares approximate solution of overdetermined Sylvester equations. SIAM J. Matrix Anal. Appl., 18(2):279-290, 1997.

[8] R. A. Horn and C. R. Johnson. Topics in Matrix Analysis. Cambridge University Press, Cambridge, 1991.

[9] B. Kågström and L. Westin. Generalized Schur methods with condition estimators for solving the generalized Sylvester equation. IEEE Trans. Automat. Control, 34(7):745-751, 1989.

[10] M. Konstantinov, V. Mehrmann, and P. Petkov. Perturbation analysis of Hamiltonian Schur and block-Schur forms. SIAM J. Matrix Anal. Appl., 23(2):387-424, 2001.

[11] V. Mehrmann and D. Watkins. Structure-preserving methods for computing eigenpairs of large sparse skew-Hamiltonian/Hamiltonian pencils. SIAM J. Sci. Comput., 22(6):1905-1925, 2000.

[12] J. M. Ortega and W. C. Rheinboldt. Iterative Solution of Nonlinear Equations in Several Variables. Academic Press, New York, 1970.

[13] J. Stefanovski and K. Trenčevski. Antisymmetric Riccati matrix equation. In 1st Congress of the Mathematicians and Computer Scientists of Macedonia (Ohrid, 1996), pages 83-92. Sojuz. Mat. Inform. Maked., Skopje, 1998.

[14] G. W. Stewart. Error bounds for approximate invariant subspaces of closed linear operators. SIAM J. Numer. Anal., 8:796-808, 1971.

[15] G. W. Stewart. Error and perturbation bounds for subspaces associated with certain eigenvalue problems. SIAM Rev., 15:727-764, 1973.

[16] G. W. Stewart and J.-G. Sun. Matrix Perturbation Theory. Academic Press, New York, 1990.

[17] F. Tisseur. A chart of backward errors for singly and doubly structured eigenvalue problems. SIAM J. Matrix Anal. Appl., 24(3):877-897, 2003.

[18] F. Tisseur and K. Meerbergen. The quadratic eigenvalue problem. SIAM Rev., 43(2):235-286, 2001.

[19] C. F. Van Loan. A symplectic method for approximating all the eigenvalues of a Hamiltonian matrix. Linear Algebra Appl., 61:233-251, 1984. 